Int. J. Morphol.,

28(1):99-103, 2010.

\title{
Medición Comparada de Procesos Odontoblásticos y Canalículos Dentinarios
}

\author{
Comparative Measurement of Odontoblastic Processes and Dentinal Tubules
}

"Alicia Kohli; ${ }^{* *}$ Graciela Garcia; ${ }^{* * *}$ Stella M. Pezzotto \& ${ }^{* * *}$ Leonor Poletto

\begin{abstract}
KOHLI, A.; GARCiA, G.; PEZzotTO, S. M. \& POLETTO, L. Medición comparada de procesos odontoblásticos y canalículos dentinarios. Int. J. Morphol., 28(1):99-103, 2010.

RESUMEN: El complejo dentinopulpar comparte el odontoblasto cuyo cuerpo está ubicado en la parte externa de la pulpa dentaria. De su polo apical se desprende el proceso odontoblástico que se introduce en el canalículo dentinal atravesando la dentina originando en su recorrido múltiples colaterales hasta su finalización. Describir el proceso fue ardua tarea de investigadores que combinaron técnicas histológicas para preservarlo en el interior de los canalículos utilizando microscopio electrónico de transmisión o scanning para visualizarlo. En un trabajo previo observamos ambos tejidos unidos por los odontoblastos y los procesos coloreados. El objetivo actual fue medir micrométricamente la longitud de procesos y canalículos para verificar si ambas son similares tanto en corona como en raíz. Se utilizaron treinta dientes sanos, extraídos por razones ortodóncicas de ambos sexos, cuyas edades oscilaron entre 6 y 18 años. Se descubrió la pulpa dental, se fijó y se dividieron en partes iguales. Las mitades sin pulpa se prepararon con técnica por desgaste. Las mitades que conservaron la pulpa se desmineralizaron aplicándoseles Colagenasa tipo II y coloreándolos con la técnica de Schmorl. Con microscopio óptico y una cuadrícula calibrada con micrómetro objetivo se midió primero el ancho de los campos histológicos y luego la longitud de procesos y canalículos dentinales. Los resultados fueron analizados con pruebas de chi2, t de Student y test exacto de Fischer con un nivel de significación del 5\%. Observamos que el ancho de los campos histológicos coloreados tuvo una retracción del $92 \%$ respecto al mismo campo en el desgaste y que la longitud de canalículos siempre fue mayor que la de procesos, si bien se hallaron cuatro coincidencias de longitudes en corona y una en raíz. Estos resultados sugieren que la longitud del proceso aún sigue siendo tema controversial. La precausión profesional en los tratamientos odontológicos es el único medio para evitar dañarlo.
\end{abstract}

PALABRAS CLAVE: Complejo dentinopulpar; Proceso odontoblástico; Técnica histológica.

\section{INTRODUCCIÓN}

El complejo dentinopulpar comparte una célula llamada odontoblasto cuyo cuerpo está ubicado en la parte más externa de la pulpa dentaria. Esta célula posee un núcleo basal, organoides necesarios para la síntesis de proteínas como el retículo endoplasmático rugoso, el aparato de Golgi y mitocondrias. De su polo apical se desprende el proceso odontoblástico, el cual se introduce en el interior del canalículo dentinal, atravesando la dentina hasta su finalización en forma ramificada, dicotómica o única. El proceso esta formado por microtúbulos y microfilamentos, vesículas recubiertas por membrana y cuerpos parecidos a lisosomas. En su recorrido origina múltiples colaterales.
Describir el proceso fue una ardua tarea de muchos investigadores. Con microscopio electrónico de transmisión o de scanning, se lo visualizó ocupando la predentina o en las cercanías del límite amelodentinario, tanto en hombres como en animales (Brännström \& Garberoglio, 1972; Grossman \& Austin, 1983; Gunji \& Kobayashi, 1983).

Yamada et al. (1983) utilizó una técnica con descalcificación y digestión parcial con colagenasa Tipo II en dentina humana. Con scanning observó las prolongaciones gruesas en predentina que disminuían de grosor por la emergencia de colaterales en la parte media de la dentina y con ramificaciones finales muy delgadas en el límite amelodentinario.

* Cátedra de Histología, Facultad de Odontología, Universidad Nacional de Rosario, Argentina.

** Facultad de Ciencias Bioquímicas, Universidad Nacional de Rosario, Argentina.

***Consejo de Investigaciones, Universidad Nacional de Rosario, Argentina. 
Sigal \& Chernecky (1988) observaron que los procesos terminaban con forma esférica en el límite amelodentinario utilizando la técnica de inmunofluorecencia en humanos y ratas.

En un estudio con microscopio confocal y scanning los hallaron nuevamente en el tercio interno de la dentina (Goracci et al., 1999) por lo cual se considera que la longitud del proceso odontoblástico sigue siendo un tema controversial.

En un trabajo previo desarollamos una técnica para dientes humanos combinando la desmineralización y eliminación de fibras colágenas, coloreando posteriormente al tejido con hemalumbre-eosina, PAS y Schmorl (Kohli et al., 2004).

Posteriormente aplicamos un método de medición para identificar la coloración que facilitara la visualización de los procesos, observando que con la de Schmorl se los reconocia hasta el borde exterior de la dentina, resultados aún no publicados. Midiendo en forma micrométrica la longitud de los procesos en dichos preparados y la longitud de los canalículos dentinales en los preparados por desgaste se podría analizar si entre ellos hay similitud de longitudes tanto en corona como en raíz, objetivo de este trabajo.

\section{MATERIAL Y MÉTODO}

Se utilizaron treinta dientes humanos, sanos, extraídos por razones ortodóncicas, de ambos sexos, cuyas edades oscilaron entre 6 y 18 años, los que luego fueron separados según sexo, edad y pieza dentaria.

Se realizó un orificio descubriendo la pulpa dental, que fue fijada in situ, se estandarizó la división del diente en dos partes iguales y se los partió. Cada mitad se observó con luz y lupa para diferenciar la mitad del diente que conservó la pulpa adherida de aquélla sin pulpa. Las mitades sin pulpa se prepararon con técnica por desgaste. Las láminas transparentes se deshidrataron, aclararon y adhirieron a los portaobjetos con bálsamo de Canadá protejidas con cubreobjetos. Las mitades que conservaron la pulpa se desmineralizaron en ácido nítrico al 8\%, aplicándoseles la técnica de colagenasa Tipo II; luego se las deshidrató, clarificó e incluyó en parafina según la técnica de rutina. Cortadas a un espesor de $5 \mu \mathrm{m}$, se desparafinaron, hidrataron y colorearon con la técnica de Schmorl.

Posteriormente, se procedió a medir con microscopio óptico el ancho de los campos histológicos elegidos, con- tando el número de cuadritos de la cuadrícula por ancho del campo, en todos los preparados a mayor aumento (400X).

La longitud del proceso odontoblástico desde su nacimiento en la célula hasta la pérdida de su visión fue medida utilizando una cuadrícula de cinco cuadritos de lado calibrada con el micrómetro objetivo a 400 aumentos, aplicando su costado como regla en la que cada cuadrito mide $260 \mu$ de largo, de manera que contando los cuadritos que mide el proceso y multiplicándolos por $260 \mu$ se obtiene la longitud final.

Los resultados fueron analizados con pruebas de $\mathrm{chi}^{2}$, $\mathrm{t}$ de Student y test exacto de Fischer con un nivel de significación del $5 \%$.

\section{RESULTADOS}

$\mathrm{Al}$ examen microscópico a menor aumento (10X), se observó que la pulpa dental permaneció unida a la corona en 18 piezas dentales, a la raíz en 6 y en otros 6 se la vió unida tanto en corona como en raíz, separándoselos en tres grupos. En el primer grupo la edad promedio fué de 14 años, la proporción de mujeres $75 \%$, predominando las piezas inferiores. En el segundo grupo la edad promedio fué de 14 años, $75 \%$ varones, mayoría de piezas superiores. En el tercer grupo el promedio de edad fue de 15 años, la proporción de mujeres $75 \%$ y mayoría de piezas dentales superiores, como se observa en la Fig. 1.

Al medir el ancho de los campos histológicos se observó que el $92 \%$ de los preparados coloreados tuvieron una retracción con respecto al mismo campo en el desgaste y sólo en $8 \%$ se mantuvo la igualdad de los mismos.

No se observaron longitudes similares en corona y raiz simultáneamente. El chi ${ }^{2}$ calculado para 1 grado de libertad (GL) al 5\%, con respecto al tabulado no fue significativo $(p=0,26)$. Como una frecuencia esperada fue menor que 5, se aplicó el test exacto de Fischer, el que tampoco fue significativo $(p=0,24)$.

La medición de la longitud de procesos se realizó en las zonas de corona (1) y de raíz (2). Cuando los procesos se visualizaron hasta el borde de la dentina coronal o radicular al número se le unió la letra $\mathrm{A}$ y la $\mathrm{B}$ si los procesos se visualizaban en un recorrido más corto. Quedando conformados los grupos en corona: $1 \mathrm{~A}$, procesos largos con 20 preparados, $1 \mathrm{~B}$, procesos cortos con 4 , y en raíz $2 \mathrm{~A}$, procesos largos con 8 y $2 \mathrm{~B}$, procesos cortos con 4 . 

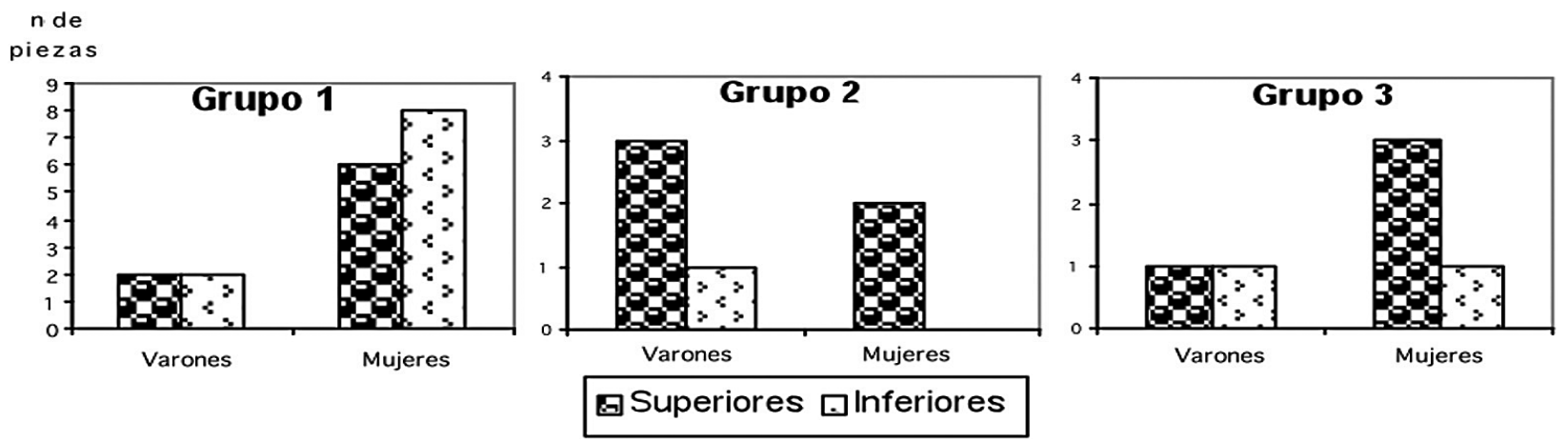

Fig. 1. Adhesión de pulpa según sexo, edad y pieza dental en los tres grupos.

En el grupo 1A, la longitud promedio de canalículos fue mayor $(26000 \mu \pm 7244,2)$ que la de procesos coloreados $(17290 \mu \pm 6723,3)$. La mediana, de mayor valor para los canalículos fue de 26000 y de 18200 para los procesos coloreados. La diferencia, cuyo valor fue de 8710 con un DS de 8014,8 . El t de la diferencia de 4,86 con 19 GL al 5\%, fue altamente significativo $(\mathrm{p}=0,0001)$, resultado similar se halló con test de Wilcoxon para muestras relacionadas $(\mathrm{p}=0,0004)$. Se comprobó en cuatro casos, marcados con asteriscos, que longitudes de canalículos y procesos fueron similares. En el grupo 1B el promedio de longitud de canalículos fue mayor $(27300 \mu \pm 4978,6)$ que la de los procesos $(9685 \mu \pm 6565,1)$. La mediana de mayor valor para los canalículos fue de 28600 y menor para los procesos 9750 . La diferencia tuvo un valor de 17615 , cuyo DS fue de 10722,9 y el t de la diferencia de 3,29 con 3 GL al 5\%, fue significativo $(\mathrm{p}=0,046)$, al igual que aplicando el test de Wilcoxon $(\mathrm{p}=0,06)$. Los resultados de las técnicas se presentan en Tabla I.
En el grupo 2A la longitud promedio de canalículos fue mayor $(19500 \mu \pm 8338,5)$ que la de procesos $(11375 \mu \pm 5141,6)$. La mediana tuvo un valor de 20800 y de 11050 respectivamente. La diferencia tuvo un valor de 8125 . El DS de la diferencia fue de 5844,8 y el t de la diferencia de 3,93 que al $5 \%$ con 7 GL fue significativo $(\mathrm{p}=0,006)$. Aplicando el test de Wilcoxon se originó un resultado similar $(\mathrm{p}=0,02)$. Se halló un caso en que ambas longitudes coinciden. En el grupo 2B el promedio de longitud de canalículos fue mayor $(18200 \mu \pm 5616,6)$ que la de procesos $(13715 \mu \pm 4414,3)$. La mediana de 16900 fue mayor para los canalículos y menor para los procesos 15210 . La diferencia tuvo un valor de 4485, cuyo DS fue de 9687,4, y el t de la diferencia de 0,93 , con $3 \mathrm{GL}$ al $5 \%$ no fue significativo $(\mathrm{p}=0,423)$, resultado semejante se obtuvo con el test de Wilcoxon $(\mathrm{p}=0,465)$. La comparación de técnicas se presenta en la Tabla II.

Tabla I. Medición de longitudes de canalículos y procesos en la corona en micras (n:5).

\begin{tabular}{|c|c|c|c|c|c|c|c|c|}
\hline \multicolumn{6}{|c|}{ GRUPO 1A } & \multicolumn{3}{|c|}{ GRUPO 1B } \\
\hline Preparado & Desgaste & Schmorl & Preparado & Desgaste & Schmorl & Preparado & Desgaste & Schmorl \\
\hline 2 & 18200 & 15600 & 16 & 31200 & 5200 & 1 & 20800 & 17420 \\
\hline 3 & 26000 & 20800 & 18 & 33800 & 18200 & 6 & 31200 & 1820 \\
\hline 5 & 36400 & 23400 & 19 & 18200 & 18200 & 21 & 31200 & 11700 \\
\hline 7 & 33800 & 26000 & 22 & 9100 & 9100 & 28 & 26000 & 7800 \\
\hline 8 & 23400 & 18200 & 23 & 26000 & 13000 & & & \\
\hline 10 & 31200 & 13000 & 25 & 31200 & 26000 & & & \\
\hline 11 & 26000 & 26000 & 26 & 20800 & 18200 & & & \\
\hline 12 & 31200 & 7800 & 27 & 31200 & 26000 & & & \\
\hline 13 & 29900 & 11700 & 29 & 23400 & 23400 & & & \\
\hline 14 & 26000 & 18200 & 30 & 13000 & 7800 & & & \\
\hline
\end{tabular}


Tabla II. Medición de longitudes de canalículos y procesos en la raíz en micras (n:5).

\begin{tabular}{|c|c|c|c|c|c|}
\hline \multirow[b]{2}{*}{ Preparado } & \multicolumn{2}{|c|}{ GRUPO 2A } & \multicolumn{3}{|c|}{ GRUPO 2B } \\
\hline & Desgaste & Schmorl & Preparado & Desgaste & S chmorl \\
\hline 1 & 20800 & 13000 & 5 & 13000 & 16900 \\
\hline 4 & 20800 & 13000 & 8 & 18200 & 16900 \\
\hline 9 & 31200 & 18200 & 17 & 15600 & 13520 \\
\hline 10 & 23400 & 5200 & 18 & 26000 & 7540 \\
\hline 13 & 18200 & 9100 & & & \\
\hline 15 & 10400 & 9100 & & & \\
\hline 20 & 5200 & 5200 & & & \\
\hline 24 & 26000 & 18200 & & & \\
\hline
\end{tabular}

\section{DISCUSIÓN}

En dientes de ratas y monos adultos utilizando tinción fluorescente de DI-I de carbocianina combinada con un nuevo procedimiento de imbición de gelatina y microscopio confocal se observó que los procesos odontoblásticos no se extendían hasta el límite amelo-dentinario, con la excepción de las etapas tempranas del desarrollo, en las que se los observaba largos y rectos en la corona y cortos y ramificados en la raíz (Byers \& Sugaya, 1995).

En nuestro trabajo con microscopio óptico se logró verlos hasta el borde del tejido, siendo los grupos $1 \mathrm{~A}$ y $2 \mathrm{~A}$ los más numerosos. Además, comprobamos la existencia de una retracción importante en los campos histológicos de los preparados teñidos, hecho que atribuímos a la pérdida de sales de calcio y fibras colágenas. La pérdida de dichos componentes podría contribuir a que los procesos ocuparan una mayor longitud de la que realmente poseen. En un estudio con Microscopio Confocal y Scannyng se los halló en el tercio interno de la dentina (Goracci et al.).

En otra investigación realizada en premolares humanos sanos de 15-20 años, extraídos por razones ortodóncicas, que fueron descalcificados, procesados para tinción fluorescente de Dil o Phalloidin-Rhodamine y observados con Microscopio Electrónico de Transmisión, se los observó no más allá del interior de la dentina (Kunihiko et al., 2002). Si bien en nuestro trabajo el rango de edad es mayor, el promedio de edad incluye una población de piezas dentales juveniles. En dientes descalcificados de ratas y monos coloreados con TRITC-phalloidin y Alexa Fluor phalloidin y Dil para microscopios Laser Confocal y de Fluorescencia, se observó que los procesos fueron visibles en zonas cuspidea y cervical hasta las 2 semanas de edad y a las 100 semanas sólo se los encontró en la cuspidea (Tsuchiya et al., 2002). Sin embargo, nuestro estudio fue realizado en dientes hu- manos con un rango de edad que incluyó la niñez y adolescencia temprana, observándoselos tanto en zona cuspídea como radicular. Con el transcurso de su vida útil los dientes soportan estímulos diversos. Entre ellos padecimiento de caries, abrasiones, uso de diversos materiales de restauración, fracturas y otras causas que por la permeabilidad dentinaria predisponen a la respuesta dentinopulpar (Mjör, 2009). En un trabajo previo hemos observado que cuando los procesos coloreados con Schmorl emergen del cuerpo del odontoblasto lo hacen recubiertos con un microgranulado que al desaparecer permite observar la continuación de los canalículos vacios o bien visualizar los procesos e incluso sus ramificaciones colaterales (Kohli et al.). Recientemente se comprobó que el número de canalículos fue mayor en la superficie interna de la dentina de caninos, premolares y terceros molares y menor en la externa (Schellenberg et al., 1992; Komabayashi et al., 2008). Esta relación la reiteramos en un trabajo previo enviado a publicación. Se pondría así de manifiesto la importancia de la unión dentinopulpar, porque si bien no todos los procesos llegan al borde externo de la dentina, su intrincada relación en el espesor de este tejido sería vital para la salud de los dientes. En nuestro trabajo la longitud de canalículos siempre fue mayor que la de procesos, hecho que atribuímos a la pérdida de sales minerales en los preparados coloreados si bien se hallaron cuatro coincidencias de longitudes en la corona y una en la raíz.

Debido a estos resultados se puede considerar que la longitud del proceso aún sigue siendo un tema controversial. Sería beneficioso lograr el diseño de un método útil estandarizado para ponerlo de manifiesto, a fin de integrar la existencia de este componente al resto de los tejidos dentales. Mientras esto no ocurra la precausión profesional es el único medio para evitar dañarlo en el transcurso de los tratamientos odontológicos. 
KOHLI, A.; GARCIA, G.; PEZZOTTO, S. M. \& POLETTO, L. Comparative Measurement of odontoblastic processes and dentinal tubules. Int. J. Morphol., 28(1):99-103, 2010.

SUMMARY: The dentin-pulp complex shares the odontoblast, whose body is located in the external part of the dental pulp. The odontoblast process emerges from the apical pole and penetrates the dentinal canaliculus through the dentine producing multiple collaterals along the way. Descriibing the process was an arduous task for researchers, who combined histological techniques to preserve it in the interior of the canaliculi using transmission electron microscopy or scanning to visualize it. In a previous work we observed both tissues joined by the odontoblasts and the stained processes. The objective now was to micrometrically measure the length of the processes and canaliculi to verify if they are similar in the crown and in the root. Thirty healthy teeth were used, extracted for orthodontic reasons, from individuals of both sexes whose ages ranged from 6 to 18. The dental pulp was uncovered and attached, and they were divided in equal parts. The halves without pulp were prepared with the erosion technique. The halves that kept the pulp were demineralized with type II collagenase and stained with the Schmorl technique. With an optical microscope and a calibrated grid with a micrometric objective the width of the histological fields was first measured and then the length of the dentinal processes and canaliculi. The results were analyzed with chi2 test, Student $t$ and Fischer's exact test with a 5\% significance level. We observed that the width of the stained histological fields had a $92 \%$ retraction compared to the same field in the erosion and that the length of the canaliculi was always greater than the length of the processes, although four coincidences in length were found in the crown and one in the root. These results suggest that the length of the process is still a controversial topic. Professional precaution in odontological treatments is the safest prevention against injuries.

KEY WORDS: Pulpodentinal complex; Odontoblastic processes; Histological technique.

\section{REFERENCIAS BIBLIOGRÁFICAS}

Brännström, M. \& Garberoglio, R. The dentinal tubules and the odontoblast processes. A Scanning Electron Microscopic study. Acta Odontol. Scand., 30(3):291-311, 1972.

Byers, M. R. \& Sugaya, A. Odontoblast processes in dentin revealed by fluorescent Di-I. J. Histochem. Cytochem., 43(2):159-68, 1995.

Goracci, G.; Mori, G. \& Baldi, M. Terminal end of the human odontoblast process: a study using SEM and confocal microscopy. Clin. Oral Investig., 3(3):126-32, 1999.

Grossman, E. S. \& Austin, J. C. Scanning Electron Microscope observations on the tubule content of freeze-fractured peripheral vervet monkey dentine (Cercopithecus Pygerythrus). Arch. Oral Biol., 28(3):279-81, 1983.

Gunji, T. \& Kobayashi, S. Distribution and organization of odontoblast processes in human dentine. Arch. Histol. Jpn., 46(2):213-9, 1983.

Kohli, A.; Postiglione, G.; Poletto, L. \& Pezzotto, S. M. Coloraciones para visualizar el proceso odontoblástico. Rev. Asoc. Odontol. Argent., 92(3):229-233, 2004.

Komabayashi, T.; Nonomura, G.; Watanabe, L. G.; Marshall, G. W. \& Marshall, S. J. Dentin tubule numerical density variations below the CEJ. J. Dent., 36(11):953-8, 2008.

Kunihiko Y, Nagako Y, Sadakazu E, Masaaki I, Hidehiro O. Odontoblast processes in human dentin revealed by fluorescence labeling and transmission electron microscopy. Histochem. Cell. Biol., 118:205-12, 2002.
Mjör, I. A. Dentin permeability: The basis for understanding pulp reactions and adhesive technology. Braz. Dent. J., 20(1):3-16, 2009.

Schellenberg, U.; Krey, G.; Bossshardt, D. \& Nair, P. N. Numerical density of dentinal tubules at the pulpal wall of human permanent premolars and third molars. J. Endod., 18(3):1049, 1992.

Sigal, M. J. \& Chernecky, R. Terminal end of the odontoblast process. J. Endod., 14(11):543-5, 1988.

Tsuchiya, M.; Sasano, Y.; Kagayama, M. \& Watanabe, M. The extent of odontoblast processes in the dentin is distinct between cusp and cervical regions during development and aging. Arch. Histol. Cytol., 65(2):179-88, 2002.

Yamada, T.; Nakamura, K.; Iwaku, M. \& Fusayama, T. The extent of the odontoblast process in normal and carious human dentine. J. Dent. Res., 62(7):798-802, 1983.

Yoshiba, K.; Yoshiba, N.; Ejiri, S.; Iwaku, M. \& Ozawa, H. Odontoblast processes in human dentin revealed by fluorescence labeling and transmission electron microscopy. Histochem. Cell Biol., 118(3):205-12, 2002.

\section{Dirección para correspondencia: \\ Alicia Kohli \\ Facultad de Odontología \\ Universidad Nacional de Rosario. \\ Pasaje Rissuto 3473 \\ Rosario 2000, Santa Fe \\ ARGENTINA}

Email: Aliciakohli2009@hotmail.com

Recibido : 23-10-2009 Aceptado: 18-01-2010 
